Proc. Indian Acad. Sci. (Math. Sci.) Vol. 113, No. 1, February 2003, pp. 87-90.

Printed in India

\title{
Some approximation theorems
}

\author{
N V RAO \\ Department of Mathematics, The University of Toledo, Toledo, Ohio 43606, USA \\ E-mail: rnagise@math.utoledo.edu
}

MS received 25 February 2002; revised 24 December 2002

Abstract. The general theme of this note is illustrated by the following theorem:

Theorem 1. Suppose $K$ is a compact set in the complex plane and 0 belongs to the boundary $\partial K$. Let $\mathscr{A}(K)$ denote the space of all functions $f$ on $K$ such that $f$ is holomorphic in a neighborhood of $K$ and $f(0)=0$. Also for any given positive integer $m$, let $\mathscr{A}(m, K)$ denote the space of all $f$ such that $f$ is holomorphic in a neighborhood of $K$ and $f(0)=f^{\prime}(0)=\cdots=f^{(m)}(0)=0$. Then $\mathscr{A}(m, K)$ is dense in $\mathscr{A}(K)$ under the supremum norm on $K$ provided that there exists a sector $W=\left\{r \mathrm{e}^{i \theta} ; 0 \leq r \leq \delta, \alpha \leq \theta \leq \beta\right\}$ such that $W \cap K=\{0\}$. (This is the well-known Poincare's external cone condition). We present various generalizations of this result in the context of higher dimensions replacing holomorphic with harmonic.

\section{Keywords.}

\section{Introduction}

Axler and Ramey (personal communication) have obtained the following interesting result: Let $L^{2}\left(S^{n}\right)$ denote the usual Lebesgue space on the unit sphere $S^{n}$ with respect to the surface area measure on $S^{n} ; x_{0}$ be a fixed point in $R^{n}$. Let $\mathscr{P}\left(x_{0}, m\right)$ denote the space of all harmonic polynomials which vanish at $x_{0}$ together with all their derivatives of order less than or equal to $m$, and $m>0$. Then

Theorem AR. $\mathscr{P}\left(x_{0}, m\right)$ is dense in $L^{2}\left(S^{n}\right)$ if and only if $\left|x_{0}\right| \geq 1$.

They also posed the following questions:

(1) Does the above result remain valid if $L^{2}\left(S^{n}\right)$ is replaced by any $L^{p}\left(S^{n}\right)$ with $p>2$ ?

(2) Could $S^{n}$ be replaced by more general surfaces?

We shall show here that the answer to the 1st question is yes. Let $\mathscr{C}\left(x_{0}, S^{n}\right)$ denote the space of all continuous functions on $S^{n}$ that vanish at $x_{0}$ and the space of all continuous functions on $S^{n}$.

Theorem 2. For any positive integer $m, \mathscr{P}\left(x_{0}, m\right)$ is dense in $\mathscr{C}\left(x_{0}, S^{n}\right)$ with the sup norm if and only if $\left|x_{0}\right| \geq 1$. When $x_{0}$ is not on the sphere, then $\mathscr{C}\left(x_{0}, S^{n}\right)$ is the same as $\mathscr{C}\left(S^{n}\right)$.

Dedicated to Prof. Ashoke Roy on his 62nd birthday. 
Remark. We shall not prove that the density fails when $\left|x_{0}\right|<1$ since it is rather obvious and we shall not explicitly deal with the case when $\left|x_{0}\right|>1$, because the proof for $\left|x_{0}\right|=1$ can be imitated without any problems.

We will derive Theorem 2 as a corollary of a more general result for which we need to introduce some more notation. Let $K$ be any compact set in $R^{n}, \partial K$ its boundary. We define a notion called ECC. (This is the well-known Poincare's external cone condition.) We say that $K$ satisfies ECC at a point $x_{0}$ if there exists a closed solid truncated cone $W$ with vertex at $x_{0}$ such that $W \cap K=\left\{x_{0}\right\}$.

It is clear that to satisfy ECC at $x_{0}, x_{0}$ must be on the boundary of $K$ and also that the set of points where $K$ satisfies ECC is dense in the boundary of $K$. In order to see this, take any point $\xi$ on the boundary of $K$ and a ball of radius $r$ with center at $\xi$, where $r$ is arbitrary and positive. There must exist a point $\eta$ outside $K$ such that $|\eta-\xi|<r / 2$ for otherwise $\xi$ would be an interior point of $K$. Now choose a nearest point to $\eta$ in $K$, say $\lambda$. Clearly $|\lambda-\eta|=\delta<r / 2$ and the ball of radius $\delta$ with center at $\eta$ is entirely contained in the ball of radius $r$ with center at $\xi$. Now $\lambda$ must belong to the boundary of $K$, must lie within a distance of $r$ from $\xi$ and satisfies ECC for $K$.

Let $\mathscr{H}\left(x_{0}, K\right)$ denote the space of all functions $f$ on $K$ such that $f$ vanishes at $x_{0}$ and is the restriction to $K$ of a function harmonic in a neighborhood of $K$. Let $\mathscr{H}\left(m, x_{0}, K\right)$ denote the space of all functions $f$ on $K$ such that $f$ is the restriction to $K$ of a function, harmonic in a neighborhood of $K$ and it, together with all its derivatives of order $\leq m$ vanish at $x_{0}$.

We shall assume the following well-known result:

Lemma A. Let $K$ be any closed ball in $R^{n}$ and $x_{0}$ belong to $K$. Then $\mathscr{P}\left(x_{0}, m\right)$ is dense in $\mathscr{H}\left(m, x_{0}, K\right)$.

Also we need

Theorem 3. Assume $K$ satisfies $\mathrm{ECC}$ at $x_{0}$. Then for any positive integer $m, \overline{\mathscr{H}\left(m, x_{0}, K\right)}$ $\supset \mathscr{H}\left(x_{0}, K\right)$ with the sup norm.

We shall supply a proof of this later.

Proof of Theorem 2. Let $K$ be the closed unit ball in $R^{n}$ and $x_{0}$ belong to $S^{n}=\partial K$. Certainly $K$ satisfies ECC at $x_{0}$. Let $f$ belong to $\mathscr{C}\left(x_{0}, S^{n}\right)$. Let $\varepsilon$ be any positive number. It is well-known that there exists a harmonic polynomial $P$ such that

$$
|f(x)-P(x)|<\varepsilon \text { on } S^{n} .
$$

Let $h(x)=P(x)-P\left(x_{0}\right)$. Then $|f(x)-h(x)| \leq|f(x)-P(x)|+\left|P\left(x_{0}\right)\right|<2 \varepsilon$ on $S^{n}$ and also $h(x)$ belongs to $\mathscr{H}\left(x_{0}, K\right)$. But by Theorem 3 , there exists a $g$ in $\mathscr{H}\left(m, x_{0}, K\right)$ such that $|h(x)-g(x)|<\varepsilon$ and so $|f(x)-g(x)|<3 \varepsilon$. This proves Theorem 2 in view of Lemma A.

Since $\mathscr{C}\left(x_{0}, S^{n}\right)$ is dense in all $L^{p}\left(S^{n}\right)$ for $0<p<\infty$, from Theorem 2 , we have

\section{COROLLARY 4.}

For any $p, 0<p<\infty$; for any positive integer $m$, and any point $x_{0}$ on $S^{n}$, the space $\mathscr{P}\left(m, x_{0}\right)$ of harmonic polynomials that vanish together with all their derivatives of order less than or equal to $m$ is dense in $L^{p}\left(S^{n}\right)$. 
Proof of Theorem 3. Let $G(x)=\ln |x|$, if $n=2$ and $|x|^{2-n}$, if $n>2$. We may assume without loss of generality that $x_{0}=0, W=\{z ;|z| \leq \rho, z /|z| \in$ a spherical cap $D\}$, and $W \cap K=\{0\}$.

Fix a $z$ outside $K$. Then $G(x-z)$ is harmonic as a function of $x$ in a neighborhood of $K$ and in a neighborhood of the origin can be expanded in an absolutely convergent power series

$$
G(x-z)=\sum a_{\alpha}(z) x^{\alpha}
$$

where $\alpha$ is a multi-index $\left(\alpha_{1}, \alpha_{2}, \ldots, \alpha_{n}\right)$ and indices are allowed to run through all nonnegative integers. Let $|\alpha|$ denote $\alpha_{1}+\alpha_{2}+\cdots+\alpha_{n}$. Further we notice that for any fixed non-negative integer $k$, the polynomial $\sum_{|\alpha|=k} a_{\alpha}(z) x^{\alpha}$ is harmonic in $x$ and for any fixed $\alpha, a_{\alpha}(z)$ is harmonic in $z$ except at the origin and if we set $z=|z| \omega$ where $\omega$ varies on the unit sphere,

$$
a_{\alpha}(z)=|z|^{2-n-|\alpha|} a_{\alpha}(\omega)
$$

We note that $a_{\alpha}(\omega)$ is real-analytic on the unit sphere. Now let

$$
G(m, x, z)=G(x-z)-\sum_{|\alpha| \leq m} a_{\alpha}(z) x^{\alpha}
$$

Clearly for any fixed $z \neq o, G(m, x, z)$ is harmonic in a neighborhood of $K$ and vanishes together with its derivatives of order less than or equal to $m$. Then if $\mu$ is any finite Borel measure on $\partial K$ orthogonal to $\mathscr{H}(m, 0, K)$, it follows that

$$
\int G(x-z) \mathrm{d} \mu(x)=\sum_{|\alpha| \leq m} a_{\alpha}(z) \int x^{\alpha} \mathrm{d} \mu(x) \quad \begin{aligned}
& \text { for all } z \text { in } \\
& \text { the complement of } K
\end{aligned}
$$

Let $b_{\alpha}$ denote $\int x^{\alpha} \mathrm{d} \mu(x)$ and $p(k, z)$ denote $\sum_{|\alpha|=k} a_{\alpha}(z) b_{\alpha}$. By (1) it follows that $p(k, z)=|z|^{2-n-k} p(k, \omega)$ and $p(k, \omega)$ is real-analytic on the unit sphere. We claim that

$$
p(k, z) \equiv 0 \quad \text { for all } k, 1 \leq k \leq m .
$$

Suppose not. Then there would exist a positive integer $l$ such that $p(j, z) \equiv 0$ for $j>l$ and $p(l, z) \neq 0$. Because $p(l, z)$ is homogeneous and is real-analytic, the set of its zeroes on the unit sphere would be a closed set without any interior. Hence there would exist sub-cone $V$ of $W$ and a positive number $\delta$ such that

$$
|p(l, z)| \geq \delta|z|^{2-n-l} \quad \text { for all } z \in V
$$

and further by choosing a sufficiently small $\beta<\rho$, we have

$$
\left|\sigma(z)=\sum_{0 \leq k \leq l} p(k, z)\right| \geq \frac{\delta}{2}|z|^{2-n-l} \quad \text { on } U=V \cap\{|z| \leq \beta\} .
$$

Choose a hyper-plane section $S$ of $U$ through the origin and integrate $\sigma(z)$ on $S$ with respect to the surface measure on it. Since $\sigma(z)$ stays away from 0 on $U$, it has the same sign everywhere and so from (4) it follows that

$$
\left|\int_{S} \sigma(z) \mathrm{d} z\right| \geq \int|\sigma(z)| \mathrm{d} z \geq \frac{\delta}{2} \int_{S}|z|^{2-n-l} \mathrm{~d} z
$$


But the last integral is infinite for $l>0$. But on the other hand $\int_{S}|G(x-z)| \mathrm{d} z$ is uniformly bounded and so $\int_{S}\left|\int G(x-z) \mathrm{d} \mu(x)\right| \mathrm{d} z$ is finite. This, (5), and (0) lead to a contradiction establishing (2). Hence

$$
\int G(x-z) \mathrm{d} \mu(x)=b_{0} G(z) \quad \text { for every } z \text { outside } K .
$$

If $v=\mu-b_{0} \delta_{0}$ where $\delta_{0}$ is the Dirac measure at the origin, (6) can be restated as

$$
\int G(x-z) \mathrm{d} v(x)=0 \quad \text { for all } z \text { outside } K .
$$

(7) implies $v$ is orthogonal to any function $f$ which is the restriction to $K$ of a function harmonic in a neighborhood of $K$. This is a rather standard Runge argument and we omit the proof. Hence for any $f$ in $\mathscr{H}(0, K), \int f(x) \mathrm{d} v(x)=\int f(x) \mathrm{d} \mu(x)-b_{0} f(0)=0$ and so $\int f(x) \mathrm{d} \mu(x)=0$. Now by Hahn-Banach, we have Theorem 3 .

Proof of Theorem 1. Fix a $z$ outside $K$ and write the Taylor formula of order $m$ for the Cauchy kernel:

$$
\frac{1}{x-z}=-\sum_{0 \leq k \leq m} \frac{x^{n}}{z^{n+1}}+\frac{x^{m+1}}{z^{m+1}(x-z)} .
$$

Let $\mu$ be any finite Borel measure on $\partial K$ such that

$$
\int f(x) \mathrm{d} \mu(x)=0 \quad \text { for any } f \in \mathscr{A}(m, K) .
$$

So $\int x^{m+1} / z^{m+1}(x-z) \mathrm{d} \mu(x)=0$ and consequently

$$
\int \frac{1}{(x-z)}=-\sum_{0 \leq k \leq m} \frac{\int x^{n} \mathrm{~d} \mu(x)}{z^{n+1}} .
$$

Let $a_{k}=\int x^{k} \mathrm{~d} \mu(x), 0 \leq k \leq m$. Arguing as in the proof of Theorem 3, we find that $a_{k}=$ $0,1 \leq k \leq m$ and $\mu-a_{0} \delta_{0}$ is orthogonal to all functions holomorphic in a neighborhood of $K$ and so to $\mathscr{A}(0, K)$. But $\delta_{0}$ is orthogonal to $\mathscr{A}(K)$ and hence follows the theorem.

\section{Conclusion}

Several problems remain. One of them is whether ECC is really necessary. Another one is what is the capacity of the set of points where the conclusion of either Theorem 3 or Theorem 1 holds in analogy with the set of regular points for the Dirichlet problem? Lastly, what would be an analogue of this Theorem 1 in the context of several complex variables? 